\title{
Altered postural stability in elderly women following a single session of head-mounted display virtual reality
}

\author{
BŁAŻEJ CIEŚLIK ${ }^{1 *}$, ANNA SERWETA², SARA FEDERICO ${ }^{3}$, JOANNA SZCZEPAŃSKA-GIERACHA ${ }^{4}$ \\ ${ }^{1}$ Faculty of Health Sciences, Jan Dlugosz University in Czestochowa, Częstochowa, Poland. \\ ${ }^{2}$ Faculty of Physiotherapy, University School of Physical Education in Wrocław, Wrocław, Poland. \\ ${ }^{3}$ Laboratory of Neurorehabilitation Technologies, Ospedale San Camillo IRCCS, Venice, Italy. \\ ${ }^{4}$ Faculty of Physiotherapy, University School of Physical Education in Wrocław, Wrocław, Poland.
}

\begin{abstract}
Purpouse: The aim of this study was to evaluate the effect of a single session of head-mounted display virtual reality on postural stability in elderly women. Methods: Forty-seven female subjects underwent a 20-minute virtual reality session. The mean age of the subjects was 70.12 years. As an immersive source, we used a relaxing virtual reality game with a head-mounted display device. The postural stability test was conducted using a Nintendo Wii force plate. Participants completed a set of three 30-s trials in which they took a quiet bipedal eyes-open stance while standing on a hard surface: before the virtual reality session, immediately after the virtual reality session, and 2 minutes after the virtual reality session. Centre of pressure parameters were analysed in the sagittal and frontal planes. Results: Analysing the results obtained immediately after the virtual reality session, significant differences were observed in almost all examined parameters. In the sagittal plane, centre of pressure path velocity increased by $10 \%(p<0.01)$ and path standard deviation by $15 \%(p<0.05)$. In the frontal plane, centre of pressure path velocity increased by $14 \%(p<0.01)$. After 2 minutes, all examined parameters showed no significant difference compared to before the virtual reality session. Conclusions: Immediately after the virtual reality session, there was an increase in almost all examined parameters. However, after 2 minutes, all examined parameters had returned to baseline. Therefore, to reduce fall risk after a virtual reality session, it is recommended that the subject spend at least 2 minutes in a sitting position.
\end{abstract}

Key words: postural stability, wavelet, virtual reality, balance, head-mounted display

\section{Introduction}

In the twenty-first century, the use of technology in the field of medicine looks promising. The utilisation of robotics, health wearables and telemedicine in general are becoming more common in clinical practice. Another form of new technology, virtual reality (VR), has become a subject of interest of researchers around the world. According to the PubMed.gov search engine, from 2000 to 2016 , there were $13 \%$ more publications on this topic, on average, each year, however, in the past four years this growth has accelerated, averaging $25 \%$ per year. The scope of applications of various types of VR is wide, from VR as an environment for functional or cognitive therapy in patients after stroke
[1], [25], [27], through to a distraction in pain management [21] and as a psychological assessment tool related to anxiety or phobias [4], [15]. Cave automated virtual environment (CAVE) systems, simulators and head-mounted displays (HMD) are most commonly used as the VR medium.

Due to its low cost, we expect that HMD will be the fastest-growing device for various types of therapy in clinical practice, but also a diagnostic purpose. It has recently been reported that VR head-mounted displays (HMDVR) can be used to assess balance in older adults [26], head movement kinematics and patterns [23], cervical spine mobility [11], or to characterize mobility impairment during gait [8]. On the clinical side, the spectrum of using VR is very wide. VR studies have demonstrated that HMDVR could enhance the

\footnotetext{
* Corresponding author: Błażej Cieślik, Faculty of Health Sciences, Jan Dlugosz University in Czestochowa, ul. Waszyngtona 4/8, 42-200 Częstochowa, Poland. Phone: +48 34378429, e-mail: b.cieslik@ujd.edu.pl

Received: October 29th, 2020

Accepted for publication: January 11th, 2021
} 
sleep quality of intensive care unit patients [13], may serve as a mirror therapy for upper limb recovery in post-stroke survivors [28], could improve balance and gait stability of older adults [7], and could be used for vestibular rehabilitation in patients with mild cognitive impairment [17].

However, the use of HMDVR in older people may carry some risk. HMDVR, which takes the form of VR goggles, transfers the subject to a virtual world, limiting visual stimuli for a specified time. Due to altering visual input after removal of the VR goggles, HMD could affect subject's gait stability [30], but also their static balance [19]. In turn, the elderly rely heavily on vision to maintain balance [9], [20]. Hence, we hypothesize, that there is a deterioration in postural stability by disturbing the visual system after a headmounted VR session. Therefore, the aim of this study was to evaluate the effect of a single session of HMDVR on postural stability in elderly women.

\section{Materials and methods}

\subsection{Participants}

The postural stability test was conducted on 47 female subjects. All participants reported very good health. The inclusion criterion was an age of 65 years or higher. Subjects were excluded from the study if they had uncorrected vision problems, a history of stroke or falls, dizziness, vertigo, acute musculoskeletal disorders, or who were taking medication that could affect balance. The study was approved by the ethics committee of the University School of Physical Education in Wrocław, Poland. The design follows the recommendations for the second phase (VR2) of clinical trials in health using virtual reality, focusing on acceptability, feasibility, tolerability, and initial clinical efficacy [2]. All participants provided written consent.

\subsection{Balance assessment}

For the postural stability test, a Nintendo Wii Balance Board (WBB) was used to register the centre of pressure $(\mathrm{CoP})$ in the sagittal plane (anterior-posterior, AP) and the frontal plane (medial-lateral, ML). A recent review showed that, despite its limitations, WBB can be used as a reliable and valid tool for assessing standing balance 5. Participants completed a set of three 30-s trials with a quiet bipedal eyes-open stance on a hard surface: before the VR session (pre), immediately after the VR session (post), and 2-minutes after the VR session (2-min post). Subjects were instructed to fix their gaze on a mark positioned at eye level on a plain wall, approximately $1 \mathrm{~m}$ away. Each participant was protected on 3 sides (front, left, right) with rails and at the back by an examiner. Data were retrieved using custom-written LabVIEW software. The sampling frequency was $100 \mathrm{~Hz}$. Outcome variables were determined using the analysis techniques contained in SeeSway (www.rehabtools.org/ seesway), an online calculator incorporating Matlab and LabVIEW software [6]. CoP measures of standard deviation (SD) and velocity were analysed in both the AP and ML planes. The outcome measures were also examined as a velocity wavelet-derived parameter at moderate $(1.56-6.25 \mathrm{~Hz})$, low $(0.39-1.56)$, very low $(0.10-0.39 \mathrm{~Hz})$ and ultra low $(<0.10 \mathrm{~Hz})$ frequencies. The ultra low frequency is believed to be associated with the visual system, very low with the vestibular system, low with cerebellar and moderate with proprioception [3], [22]. Higher scores in individual bandwidth reflect more body sway. In order to determine the percentage involvement of a given system in the postural stability recovery process, Clark et al. [22] proposed converting individual bandwidth results into percentages. Therefore, frequency band measures are presented as percentages.

\subsection{Virtual reality immersive intervention}

The VIVE device, developed by the HTC company, was used as the display device for the HMDVR. As an immersive source, each participant took part in a relaxing VR game, in which they were in a virtual garden for 20 minutes. The whole session was conducted in a sitting position. During that time, using a controller, the subject had the task of colouring a simple picture. During the session, relaxing music was played.

\subsection{Statistical analysis}

Data were analysed using Statistica 12 software. Descriptive statistics for characterising the study population are presented with the mean and standard deviation. Data distribution was assessed using the Shapiro-Wilk test, and, due to the normal distribution, parametric tests were used. Repeated-measures analysis of variance (ANOVA) with Bonferroni post hoc test was used to compare the results obtained in each test. A significance level of $\alpha<0.05$ was established. 


\section{Results}

In Table 1, the baseline characteristics of all individuals are shown. The mean age of participants was $70.12(S D$ 6.09) years and mean body mass index (BMI) was $29.86(S D 3.47) \mathrm{kg} / \mathrm{m} 2$.

Table 1. Participants characteristics

\begin{tabular}{|l|c|}
\hline \multicolumn{1}{|c|}{ Variable } & Summary \\
\hline $\mathrm{N}$ & 47 \\
\hline Age [years], mean $(S D)$ & $70.12(6.09)$ \\
\hline Height [cm], mean $(S D)$ & $160.06(8.41)$ \\
\hline Body mass [kg], mean $(S D)$ & $76.19(7.39)$ \\
\hline BMI [kg/cm $\left.{ }^{2}\right]$, mean $(S D)$ & $29.86(3.47)$ \\
\hline$\quad$ Normal $(B M I ~ 18.5-24.9), \mathrm{n} \mathrm{[ \% ]}$ & $0(0.00)$ \\
\hline \multicolumn{1}{|c|}{ Overweight $(\mathrm{BMI} 25-29.9), \mathrm{n}[\%]$} & $32(68.08)$ \\
\hline \multicolumn{1}{|c|}{ Obese $(\mathrm{BMI}>30), \mathrm{n}[\%]$} & $15(31.92)$ \\
\hline Systolic BP [mmHg], mean $(S D)$ & $140.32(22.97)$ \\
\hline Diastolic BP [mmHg], mean $(S D)$ & $76.28(4.74)$ \\
\hline Hypertension, $\mathrm{n}[\%]$ & $15(31.92)$ \\
\hline Smokers, $\mathrm{n}[\%]$ & $5(10.63)$ \\
\hline
\end{tabular}

BMI - body mass index; BP - blood pressure; SD - standard deviation.

Mean differences with confidence interval (CI) for the $\mathrm{CoP}$ path velocity and path $\mathrm{SD}$ are provided in Table 2. Regarding the path velocity results, statisti- cally significant differences were observed in both planes $(\mathrm{AP}, F(2,92)=6.70, p=0.001$; ML, $F(2,92)$ $=8.00, p=0.0006)$. In the sagittal plane, after the VR session, participants obtained a result that was by $10.23 \%$ greater than the pre value $(0.88 S D 0.26$ pre vs. $0.97 S D 0.30$ post), while in the frontal plane the difference was $13.95 \%$ (0.43 SD 0.14 pre vs. $0.49 S D$ 0.16 post). Considering the CoP path SD results, a statistically significant difference was observed in the AP plane $(F(2,92)=5.32, p=0.006)$. The result obtained after the VR session was by $15.56 \%$ greater than the pre value $(0.45 S D 0.13$ vs. $0.52 S D 0.19)$.

As shown in Table 3, the wavelet-derived percentage velocity signal in CoP measures after the VR session represented by the ultra low-frequency bandwidth $(<0.10 \mathrm{~Hz})$ was by $23 \%$ and by $26 \%$ lower in the AP and ML planes, respectively. In the very low-frequency bandwidth $(0.10-0.39 \mathrm{~Hz})$, the CoP measures after the session increased by $8 \%$ in the AP plane, and an increase by $26 \%$ was observed in the ML plane in the lowfrequency bandwidth $(0.39-1.56 \mathrm{~Hz})$.

\section{Discussion}

Our study explored the effects of a single HMDVR session on postural stability in elderly women. For this

Table 2. VR session effects, mean differences; mean percentage difference (95\% CI; $p$-value)

\begin{tabular}{|c|c|c|}
\hline & $\mathrm{A} / \mathrm{P}$ & $\mathrm{M} / \mathrm{L}$ \\
\hline \multicolumn{3}{|l|}{ Velocity $[\mathrm{cm} / \mathrm{s}]$} \\
\hline Pre vs. post & $-0.09 ;-10.23 \%(-0.16--0.03 ;<0.01)$ & $-0.06 ;-13.95 \%(-0.10--0.01 ;<0.01)$ \\
\hline Pre vs. 2-min post & $-0.01 ;-\mathbf{1 . 1 3 \%}(-0.08-0.05 ; \mathrm{NS})$ & $0.01 ; \mathbf{2 . 3 3 \%}(-0.04-0.05 ; \mathrm{NS})$ \\
\hline Post vs. 2-min post & $0.08 ; 8.99 \%(0.01-0.14 ; 0.01)$ & $0.07 ; \mathbf{1 6 . 6 7 \%}(0.02-0.12 ;<0.01)$ \\
\hline \multicolumn{3}{|l|}{$S D[\mathrm{~cm}]$} \\
\hline Pre vs. post & $-0.07 ;-\mathbf{1 5 . 5 6 \%}(-0.13--0.00 ; 0.03)$ & $-0.04 ;-\mathbf{1 5 . 3 8 \%}(-0.09-0.01 ; \mathrm{NS})$ \\
\hline Pre vs. 2-min post & $-0.01 ;-\mathbf{2 . 2 2 \%}(-0.05-0.07 ; \mathrm{NS})$ & $-0.02 ;-7.69 \%(-0.06-0.04 ; \mathrm{NS})$ \\
\hline Post vs. 2-min post & $0.08 ; \mathbf{1 8 . 1 8 \%}(0.01-0.14 ; 0.01)$ & $0.02 ; \mathbf{7 . 1 4 \%}(-0.03-0.08 ; \mathrm{NS})$ \\
\hline
\end{tabular}

$\mathrm{A} / \mathrm{P}$ - anteroposterior, $\mathrm{M} / \mathrm{L}$ - mediolateral, NS - non-significant, $p<0.05$.

Table 3. Frequency mean values $(S D)$ of velocity wavelet analysis in sagittal and medial plane - percentage the proportion of individual component of each frequency band

\begin{tabular}{|l|c|c|c|c|c|c|c|c|}
\hline & \multicolumn{4}{|c|}{$\mathrm{A} / \mathrm{P}$} & \multicolumn{4}{c|}{$\mathrm{M} / \mathrm{L}$} \\
\cline { 2 - 10 } & $1.56-6.25 \mathrm{~Hz}$ & $0.39-1.56 \mathrm{~Hz}$ & $0.10-0.39 \mathrm{~Hz}$ & $<0.10 \mathrm{~Hz}$ & $1.56-6.25 \mathrm{~Hz}$ & $0.39-1.56 \mathrm{~Hz}$ & $0.10-0.39 \mathrm{~Hz}$ & $<0.10 \mathrm{~Hz}$ \\
\hline Pre & $36.48(4.77)$ & $41.90(3.98)$ & $15.71(5.04)$ & $5.89(1.01)$ & $37.13(6.11)$ & $39.63(5.04)$ & $17.99(7.52)$ & $5.22(1.77)$ \\
\hline Post & $36.67(6.50)$ & $41.75(5.04)$ & $17.02^{*}(4.50)$ & $4.54^{*}(1.54)$ & $35.39(5.11)$ & $41.91^{*}(5.51)$ & $18.80(6.71)$ & $3.85^{*}(1.28)$ \\
\hline 2-min post & $36.79(6.31)$ & $40.57(4.98)$ & $17.39^{*}(5.39)$ & $5.23^{\wedge}(1.49)$ & $36.07(5.34)$ & $38.82^{\wedge}(4.78)$ & $19.13(6.79)$ & $5.98^{\wedge}(1.98)$ \\
\hline$p$-value & $\mathrm{NS}$ & $\mathrm{NS}$ & 0.04 & $<0.00$ & $\mathrm{NS}$ & $<0.00$ & $\mathrm{NS}$ & $<0.00$ \\
\hline
\end{tabular}

* - statistically significant results in comparison to test 1 according to Bonferroni post hoc, $\mathrm{A} / \mathrm{P}$ - anteroposterior, $\mathrm{M} / \mathrm{L}$ - mediolateral, NS - non-significant, $p<0.05$. 
purpose, we used traditional CoP parameters as well as wavelet-based parameters. The $\mathrm{CoP}$ variability, represented by the standard deviation of postural sway, is a widely accepted measure of postural performance. In this study, we found around 15\% difference in COP variability in the sagittal plane immediately after the HMDVR session. In a 2017 study comparing fallers and non-fallers, the difference in the RMS parameter (which is equivalent to the $S D$ parameter) in the sagittal plane was just 15\% [9]. In the current study, the mean velocity results in both planes immediately after the VR session were over $10 \%$ higher than the results obtained in the pre-intervention test. It has been shown that a higher mean velocity, especially in the ML plane, can increase the risk of falling among older people [12], [16]. Such deterioration may lead to a significant increase in the risk of falling after a HMDVR session. Similar results were obtained by examining differences in postural stability during tests in real and virtual environments. The authors demonstrated that the influence of virtual environment alters postural stability and is similar to measurements conducted in trials with eyes closed [10], [18]. In 2016, Robert et al. [24], in a study assessing the effect of HMDVR in young adults, concluded that, due to sensorimotor conflict, HMDVR could have a detrimental impact on dynamic balance.

Wavelet-based parameter analysis can facilitate the interpretation of the obtained results. In both planes, a statistically significant decrease in weighting of the $<0.10 \mathrm{~Hz}$ bandwidth (associated with the visual system) was demonstrated. In the AP plane, this decrease was compensated for by increasing weighting in the $0.10-0.39 \mathrm{~Hz}$ bandwidth (vestibular system), and in the ML plane this compensation occurred at the $0.39-1.56 \mathrm{~Hz}$ bandwidth (cerebellar). Decreased weighting on visual input also occurs in eyes-closed trials [3]. We hypothesise that the decreased weighting in the ultra low bandwidth was due to altered visual input induced by the VR goggles, which did not manage to return to its initial state. The central nervous system re-weighs the available sensory information to recruit the vestibular and cerebellar systems to achieve postural stability in the AP and ML planes, respectively. The whole VR headset weighs about $500 \mathrm{~g}$, and most of that mass is on the face side. The VR session lasted 20 minutes; therefore, to stabilise the head, isometric contraction from the cervical extensors was needed. This could lead to muscle fatigue. A 2014 study demonstrated that neck musculature fatigue increased weighting in the ultra low bandwidth [14]. In our study, the visual system was altered, hence, the mechanism to achieve effective maintenance of balance could be enhanced activation of the vestibular and cerebellar systems. In a recent study, Wodarski et al. [29] performed the analysis of the energy using the wavelet decomposition during the application of HMDVR. The study demonstrated a statistically significant difference in individual frequency bands, of which the increase in the low bandwidth in ML direction exactly coincides with our study. Perhaps this change was captured in our study in the stability trial immediately after the VR session. However, in this study, the authors reported data for each signal from decomposition, and in our study, the signals are combined into a particular frequency bandwidth, which makes it difficult to directly compare the results.

Our findings have both research and clinical implications. In this study, all examined parameters returned to baseline after 2 minutes. Therefore, when using HMDVR in clinical practice, we recommend that the subject spend at least 2 minutes in a sitting position immediately after the VR session to reduce their risk of falling. Furthermore, when conducting research using HMDVR as a stability training source, it is worth bearing in mind that time can affect the results, and that measuring balance too quickly may give distorted results.

Several limitations of this study should be noted. Firstly, our study used a repeated-measures design, and in order to capture the immediate effect we used only one measure in each trial, therefore, the results are more prone to errors. Secondly, we examined a relatively small sample of patients in a single, eyes-open static condition. Third, there is no control condition in this study. This must be taken into account when interpreting the data. Additional studies are needed to help define best practices in HMDVR utilisation and to explore new therapeutic uses for VR in clinical practice.

In conclusion, our study demonstrated that almost all tested parameters were increased immediately after the VR session. At this point, the examined person uses less of their visual system, recruiting the vestibular system in the sagittal plane and the cerebellum in the frontal plane to a greater extent. After 2 minutes, however, the examined parameters had returned to baseline. To reduce the fall risk after a VR session, it is recommended that the subject spend at least 2 minutes in a sitting position.

\section{Funding}

This research did not receive any specific grant from funding agencies in the public, commercial, or not-for-profit sectors. 


\section{References}

[1] Bartnicka J., Herrera C., Michnik R., Pavan E.E., VERCESI P., VARELA-DONOSO E., GARRIDO D., The Role of Virtual Reality and Biomechanical Technologies in Stroke Rehabilitation, [in:] S. Nazir, A.M. Teperi, A. Polak-Sopińska (eds.), Advances in Human Factors in Training, Education, and Learning Sciences, Advances in Intelligent Systems and Computing, Springer International Publishing, 2018.

[2] Birckhead B., Khalil C., LI X., Conovitz S., Rizzo A., DANovitch I., Bullock K., SPIEgel B., Recommendations for Methodology of Virtual Reality Clinical Trials in Health Care by an International Working Group: Iterative Study, JMIR Mental Health, 2019, 6, e11973.

[3] Chagdes J.R., Rietdyk S., Haddad J.M., Zelaznik H.N., Raman A., Rhea C.K., Silver T.A., Multiple Timescales in Postural Dynamics Associated with Vision and a Secondary Task Are Revealed by Wavelet Analysis, Exp. Brain. Res., 2009, 197, 297-310.

[4] CieŚlik B., MazureK J., RutKowski S., KiPER P., Turolla A., SzCZePańsKa-Gieracha J., Virtual Reality in Psychiatric Disorders: A Systematic Review of Reviews, Complementary Therapies in Medicine, 2020, 52, 102480.

[5] Clark R.A., Mentiplay B.F., Pua Y.-H., Bower K.J., Reliability and Validity of the Wii Balance Board for Assessment of Standing Balance: A Systematic Review, Gait Posture, 2018, 61, 40-54.

[6] Clark R.A., PuA Y.-H., See Sway - A Free Web-Based System for Analysing and Exploring Standing Balance Data, Computer Methods and Programs in Biomedicine, 2018, 159, 31-36.

[7] Delgado F., Der Ananian C., The Use of Virtual Reality through Head-Mounted Display on Balance and Gait in Older Adults: A Scoping Review, Games Health J., 2020.

[8] Gérin-Lajoie M., Ciombor D.M.C.K., Warren W.H., AARON R.K., Using Ambulatory Virtual Environments for the Assessment of Functional Gait Impairment: A Proof-of-Concept Study, Gait Posture, 2010, 31, 533-536.

[9] Howcroft J., Lemaire E.D., Kofman J., McIlroy W.E., Elderly Fall Risk Prediction Using Static Posturography, PLoS One, 2017, 12 (2), e0172398.

[10] Jurkojć J., Wodarski P., Michnik R., Bieniek A., Herrera Ligero C., PAVAn E., The Influence of $3 D$ Virtual Sceneries on Ability to Maintain Balance, 24th International Conference Engineering Mechanics, 2018, 341-344.

[11] Kiper P., Baba A., Alhelou M., Pregnolato G., Maistrello L., Agostini M., Turolla A., Assessment of the Cervical Spine Mobility by Immersive and Non-Immersive Virtual Reality, Journal of Electromyography and Kinesiology, 2020, 51, 102397.

[12] Kwok B.-C., Clark R.A., Pua Y.-H., Novel Use of the Wii Balance Board to Prospectively Predict Falls in CommunityDwelling Older Adults, Clin. Biomech. (Bristol, Avon), 2015, 30, 481-484.

[13] LeE S.Y., KANG J., Effect of Virtual Reality Meditation on Sleep Quality of Intensive Care Unit Patients: A Randomised Controlled Trial, Intensive Crit. Care Nurs., 2020, 59, 102849.

[14] Liang Z., Clark R., Bryant A., QueK J., PuA Y.H., Neck Musculature Fatigue Affects Specific Frequency Bands of Postural Dynamics during Quiet Standing, Gait Posture, 2014, 39, 397-403.

[15] MAPles-Keller J.L., BunNell B.E., Kim S.-J., RothBAum B.O., The Use of Virtual Reality Technology in the Treatment of
Anxiety and Other Psychiatric Disorders, Harv. Rev. Psychiatry, 2017, 25, 103-113.

[16] Merlo A., Zemp D., Zanda E., Rocchi S., Meroni F., TetTamanti M., Recchia A., Lucca U., Quadri P., Postural Stability and History of Falls in Cognitively Able Older Adults: The Canton Ticino Study, Gait Posture, 2012, 36, 662-666.

[17] Micarelli A., Viziano A., Micarelli B., Augimeri I., AlESSANDRINI M., Vestibular Rehabilitation in Older Adults with and without Mild Cognitive Impairment: Effects of Virtual Reality Using a Head-Mounted Display, Arch. Gerontol. Geriatr., 2019, 83, 246-256.

[18] Michnik R., Jurkojć J., Wodarski P., Gzik M., BienieK A., The Influence of the Scenery and the Amplitude of Visual Disturbances in the Virtual Reality on the Maintaining the Balance, Arch. Budo., 2014, 10, 133-140.

[19] MichNiK R., JURKOJĆ J., WODARSKI P., GZIK M., JOCHYMCZYK-WoźNIAK K., BIENIEK A., The Influence of Frequency of Visual Disorders on Stabilographic Parameters, Acta Bioeng. Biomech., 2016, 18, 25-33.

[20] Osoba M.Y., RaO A.K., Agrawal S.K., Lalwani A.K., Balance and Gait in the Elderly: A Contemporary Review, Laryngoscope Investig. Otolaryngol., 2019, 4, 143-153.

[21] Pourmand A., Davis S., Lee D., Barber S., SikKa N., Emerging Utility of Virtual Reality as a Multidisciplinary Tool in Clinical Medicine, Games Health J., 2017, 6, 263-270.

[22] Quek J., Brauer S.G., Clark R., Treleaven J., New Insights into Neck-Pain-Related Postural Control Using Measures of Signal Frequency and Complexity in Older Adults, Gait Posture, 2014, 39, 1069-1073.

[23] Quinlivan B., Butler J.S., Beiser I., Williams L., McGovern E., O'Riordan S., Hutchinson M., ReIlly R.B., Application of Virtual Reality Head Mounted Display for Investigation of Movement: A Novel Effect of Orientation of Attention, J. Neural. Eng., 2016, 13, 056006.

[24] Robert M.T., Ballaz L., Lemay M., The Effect of Viewing a Virtual Environment through a Head-Mounted Display on Balance, Gait Posture, 2016, 48, 261-266.

[25] Rutkowski S., Kiper P., Cacciante L., Cieślik B., MaZureK J., Turolla A., SzczepańsKa-Gieracha J., Use of Virtual Reality-Based Training in Different Fields of Rehabilitation: A Systematic Review and Meta-Analysis, J. Rehabil. Med., 2020, 52 (11), jrm00121.

[26] Saldana S.J., Marsh A.P., ReJeski W.J., Haberl J.K., Wu P., Rosenthal S., IP E.H. Assessing Balance through the Use of a Low-Cost Head-Mounted Display in Older Adults: A Pilot Study, Clin. Interv. Aging., 2017, 12, 1363-1370.

[27] SzCZEPańsKa-Gieracha J., CieŚlik B., RutKowski S., Kiper P., Turolla A., What Can Virtual Reality Offer to Stroke Patients? A Narrative Review of the Literature, Neuro Rehabilitation, 2020, 47, 109-120.

[28] Weber L.M., Nilsen D.M., Gillen G., Yoon J., Stein J., Immersive Virtual Reality Mirror Therapy for Upper Limb Recovery After Stroke: A Pilot Study, Am. J. Phys. Med. Rehabil., 2019, 98, 783-788.

[29] Wodarski P., Jurkojć J., Gzik, M., Wavelet Decomposition in Analysis of Impact of Virtual Reality Head Mounted Display Systems on Postural Stability, Sensors, 2020, 20, 7138.

[30] Wodarski P., Jurkojć J., Polechoński J., Bieniek A., Chrzan M., Michnik R., GziK M., Assessment of Gait Stability and Preferred Walking Speed in Virtual Reality, Acta Bioeng. Biomech., 2020, 22, 127-134. 